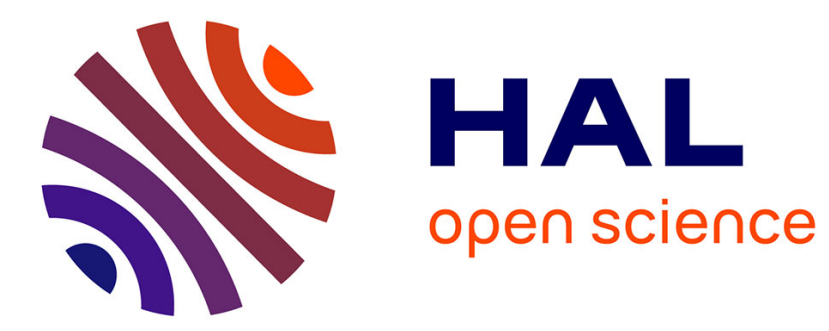

\title{
Spatiotemporal solitons in the Ginzburg-Landau model with a two-dimensional transverse grating
}

Dumitru Mihalache, D. Mazilu, F. Lederer, Hervé Leblond, Boris Malomed

\section{To cite this version:}

Dumitru Mihalache, D. Mazilu, F. Lederer, Hervé Leblond, Boris Malomed. Spatiotemporal solitons in the Ginzburg-Landau model with a two-dimensional transverse grating. Physical Review A : Atomic, molecular, and optical physics [1990-2015], 2010, 81 (2), pp.025801. 10.1103/PhysRevA.81.025801. hal-03187676

\section{HAL Id: hal-03187676 \\ https://univ-angers.hal.science/hal-03187676}

Submitted on 1 Apr 2021

HAL is a multi-disciplinary open access archive for the deposit and dissemination of scientific research documents, whether they are published or not. The documents may come from teaching and research institutions in France or abroad, or from public or private research centers.
L'archive ouverte pluridisciplinaire HAL, est destinée au dépôt et à la diffusion de documents scientifiques de niveau recherche, publiés ou non, émanant des établissements d'enseignement et de recherche français ou étrangers, des laboratoires publics ou privés. 


\title{
Spatiotemporal solitons in the Ginzburg-Landau model with a two-dimensional transverse grating
}

\author{
D. Mihalache, ${ }^{1}$ D. Mazilu, ${ }^{1}$ F. Lederer, ${ }^{2}$ H. Leblond,${ }^{3}$ and B. A. Malomed ${ }^{4}$ \\ ${ }^{1}$ Horia Hulubei National Institute for Physics and Nuclear Engineering (IFIN-HH), 407 Atomistilor, \\ Magurele-Bucharest, R-077125, Romania \\ ${ }^{2}$ Institute of Solid State Theory and Theoretical Optics, Friedrich-Schiller Universität Jena, Max-Wien-Platz, 1, D-077743 Jena, Germany \\ ${ }^{3}$ Laboratoire POMA, CNRS FRE 2988, Université d'Angers, 2 Bd Lavoisier, F-49045 Angers Cedex 01, France \\ ${ }^{4}$ Department of Physical Electronics, Faculty of Engineering, Tel Aviv University, Tel Aviv Il-69978, Israel
} (Received 27 August 2009; published 12 February 2010)

\begin{abstract}
We explore families of spatiotemporal dissipative solitons in a model of three-dimensional (3D) laser cavities including a combination of gain, saturable absorption, and transverse grating. The model is based on the complex Ginzburg-Landau equation with the cubic-quintic nonlinearity and a two-dimensional (2D) periodic potential representing the grating. Fundamental and vortical solitons are found in a numerical form as attractors in this model and their stability against strong random perturbations is tested by direct simulations. The fundamental solitons are completely stable while the vortices, built as rhombus-shaped complexes of four fundamental solitons, may be split by perturbations into their constituents separating in the temporal direction. Nevertheless, a sufficiently strong grating makes the vortices practically stable objects.
\end{abstract}

DOI: 10.1103/PhysRevA.81.025801

PACS number(s): 42.65.Tg, 42.65.Sf, 47.20.Ky

The complex Ginzburg-Landau (CGL) equation [1,2] is a generic model for pattern-forming transitions in various dissipative media such as optical cavities, viscous fluid flows and thermal convection, reaction-diffusion mixtures, and so on. The characteristic feature of the CGL equations in situations when they are supposed to produce stable localized pulses (dissipative solitons, DS's [3,4]) is the cubic-quintic (CQ) combination of nonlinear gain and loss added to the usual linear loss term [1]. In photonics, this combination corresponds (at least at the phenomenological level) to the interplay of the linear gain and saturable absorption [2]. Optical media that feature the CQ nonlinearity in the effective index of refraction include chalcogenide glasses [5], organic materials [6], colloids [7], dye solutions [8], and ferroelectrics [9]. It was also predicted that such nonlinearities can be synthesized by means of the cascading mechanism [10]. Solitary-pulse solutions and their stability were first investigated in detail in the one-dimensional (1D) version of the CQ CGL equation [11]. A challenging problem is the search for stable twodimensional (2D) and three-dimensional (3D) DS's as well as localized modes with embedded vorticity (i.e., vortex solitons). As concerns solitons of the latter type, a fundamental difficulty is that they are vulnerable to azimuthal perturbations, which tend to split them into sets of zero-vorticity fragments [12-14]. Actually, the CQ CGL equation was originally introduced by Petviashvili and Sergeev [15] in the 2D form with the objective of generating such vortices. Stable 2D dissipative vortex rings in the form of "spiral solitons" with topological charges (vorticity) $S=1$ and 2 were found in the framework of the CQ CGL equation in Ref. [16]. Stable fundamental $(S=0)$ DS's are known in 3D models based on CQ CGL equations [17-20]. Three-dimensional double-soliton complexes including rotating ones [21] were also found. Three-dimensional stable vortex solitons, otherwise known as vortex tori, with $S=1,2$, and 3 were reported as solutions to the CQ CGL equation in Ref. [22]. Their stability was analyzed in terms of the growth rates of perturbation eigenmodes and verified in direct simulations. It is also relevant to mention that the dynamics of weakly nonstationary 2D and 3D soliton complexes were studied in different conservative and dissipative settings in Refs. [23-28]. Collisions between coaxial 3D vortex solitons in the framework of the CQ CGL equation were investigated in Ref. [29].

In terms of the light propagation in bulk media along coordinate $z$, the scaled form of the 3D CQ CGL equation for the amplitude $U(x, y, z, t)$ of the electromagnetic field is [22]

$$
\begin{aligned}
& i U_{z}+(1 / 2)\left(U_{x x}+U_{y y}\right)+(D / 2) U_{t t} \\
& \quad+\left[i \delta+(1-i \varepsilon)|U|^{2}-(v-i \mu)|U|^{4}\right] U=0,
\end{aligned}
$$

where $(x, y)$ and $t$ are the transverse coordinates and reduced temporal variable. The coefficients that are scaled to be $1 / 2$ and 1 in Eq. (1) account, respectively, for the diffraction in the transverse plane and self-focusing Kerr nonlinearity, $v$ is the coefficient of the quintic self-focusing or defocusing, and $D$ is the group-velocity dispersion (GVD), with $D>0 / D<0$ corresponding to the anomalous or normal GVD. In the dissipative part of the equation, real constants $\delta, \varepsilon$, and $\mu$ represent, respectively, the linear loss, cubic gain, and quintic loss. The dispersion of the linear loss (spectral filtering) is not included in Eq. (1) as it is not essential to the stability of multidimensional solitons and is negligible in physically relevant models $[22,30]$. In the conservative counterpart of the CQ CGL equation (i.e., the nonlinear Schrödinger equation with the CQ nonlinearity) the quintic term must be selfdefocusing (in both 2D and 3D settings), which corresponds to $v>0$ in Eq. (1) to arrest the collapse driven by the self-focusing cubic nonlinearity $[12,14]$. However, it was shown in Ref. [22] that multidimensional DS's may remain stable with the self-focusing quintic term $(v<0)$; the collapse being arrested by the action of the quintic dissipation. On the contrary, vortex solitons cannot be stable against splitting in the framework of Eq. (1) and its 2D counterpart without adding an extra term $-i \beta\left(U_{x x}+U_{y y}\right)$ with $\beta>0$, which will account for an effective diffusivity in the transverse plane [22]. However, the diffusivity does not emerge in laser-cavity models except for some special cases [31]. Therefore, an important issue is 
to find a physically relevant modification of the $2 \mathrm{D}$ and $3 \mathrm{D}$ CGL models that will support stable localized vortices. In the framework of the 2D setting, it was recently reported that this is possible if a 2D lattice (spatially periodic potential) that represents a transverse grating created in the laser cavity is added to the model [30]. The grating may be a permanent one created by means of the diffusion technology [32] or a virtual grating induced in a photorefractive material [33]. The splitting instability of 2D vortex complexes, built as sets of four peaks pinned by the transverse periodic potential, may be completely suppressed by the lattice potential [30].

The objective of the present work is to study the existence and stability of both fundamental and vortical 3D spatiotemporal DS's in the model of the bulk optical medium with the anomalous GVD $D=+1$, which contains the 2D transverse grating as the stabilizing element. The accordingly modified version of Eq. (1) is

$$
\begin{gathered}
i U_{z}+(1 / 2)\left(U_{x x}+U_{y y}+U_{t t}\right)+\left\{i \delta+(1-i \varepsilon)|U|^{2}\right. \\
\left.-(v-i \mu)|U|^{4}+p[\cos (2 x)+\cos (2 y)]\right\} U=0,
\end{gathered}
$$

where $p$ is the strength of the respective lattice potential, the period of which is scaled to be $\pi$. Taking into regard the rescalings used to derive Eq. (2) from the full wavepropagation equation and assuming that the wavelength of light and the realistic value of the grating's period are, respectively, $\lambda \sim 1 \mu \mathrm{m}$ and $\Lambda \sim 30 \lambda$, one can conclude that $z=1$ and $t=1$ correspond in physical units to $\sim 1 \mathrm{~mm}$ and $10 \mathrm{fs}$ and $p=1$ corresponds to the amplitude of the local modulation of the refractive index $\delta n \sim 0.001$. Stationary solutions were generated as attractors of Eq. (2) by direct simulations of this equation. Thus found objects are single-peaked fundamental solitons (with $S=0$ ) and rhombus-shaped vortical solitons (onsite vortices), which are built as complexes of four peaks of the local intensity, set at local minima of the lattice potential with a nearly empty site in the middle, see Fig. 3. The topological charge (vorticity) of the complex is provided by the phase shifts of $\pi / 2$ between adjacent peaks, which corresponds to the total phase circulation of $2 \pi$ around the core of the pattern as it should be in vortices with $S=1$. The numerical results are presented in the following for a fixed set of three parameters in Eq. (2): $\mu=1, v=0.1$, and $\delta=0.4$, which adequately represent the generic case while cubic gain $\varepsilon$ and strength $p$ of the transverse periodic potential are varied.
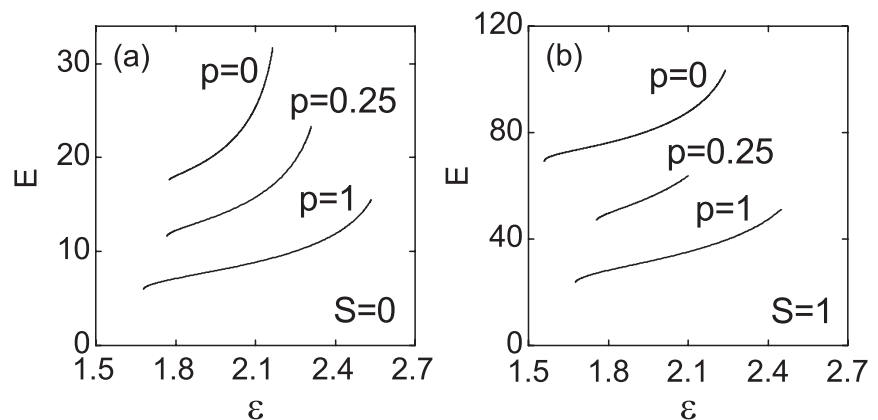

FIG. 1. (a) Total energy $E$ versus cubic gain $\varepsilon$ for fundamental solitons and (b) for rhombus-shaped vortices with $S=1$ at different fixed values of strength $p$ of the lattice potential. Note the difference in the scale on the vertical axes in the two panels. (a)

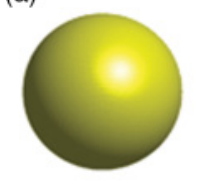

(b)

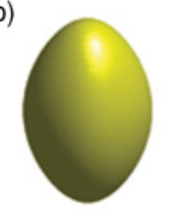

(c)

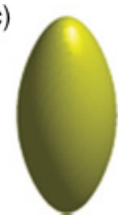

FIG. 2. (Color online) Isosurface plots of total intensity $|U(x, y, t)|^{2}$ showing typical stable fundamental solitons: (a) $p=0$, (b) $p=1$, and (c) $p=4$. Here $\varepsilon=2$. Each soliton occupies, approximately, one cell of the transverse grating, its temporal extension being $T \simeq 2$ (cf. Fig. 5).

As the shape and stability of the DS's are most sensitive to values of these coefficients they can be easily adjusted in the experiment. The simulations of Eq. (2) were run using a 3D Crank-Nicolson finite-difference scheme with typical transverse and longitudinal step sizes $\Delta x=\Delta y=\Delta t=0.05$ and $\Delta z=0.005$. In most cases, we used 281 discretization points for $t$ and $161 \times 161$ mesh points in the $(x, y)$ plane for the fundamental solitons or $201 \times 201$ points for the rhombic vortices. After a particular stationary solution was found from the direct integration of Eq. (2), it was then used as the input for a new run of simulations with slightly modified values of the parameters; the aim of this was to find an attractor corresponding to these new values. When the simulations converged to localized modes their stability was additionally tested by adding to them white noise at the amplitude level of up to $10 \%$ and running the subsequent simulations-typically, up to $z=500$ for fundamental solitons and up to $z=3000$ for vortices [as follows from Eq. (2), $z=500$ corresponds to $\sim 25$ diffraction lengths defined by the grating]. To make conclusions about the stability we monitored the evolution of amplitude $\max |U(x, y, t)|$ of the pattern and the total energy of the solution $E=\iiint|U(x, y, z, t)|^{2} d x d y d t$. The perturbed solution was identified as a stable one if its amplitude and shape will relax back to the unperturbed values. When localized states cannot self-trap in the course of the evolution or existed temporarily, but eventually turned out to be unstable, the eventual state of the system will be either $U=0$ or a quasirandom speckle pattern covering the entire domain. The decay toward zero was observed when the gain was too weak versus the dissipation [i.e., $\varepsilon$ in Eq. (2) was too small]. In the opposite case with $\varepsilon$ too large, the speckle structure was filling out all lattice cells, expanding also along $t$.

In Fig. 1 we show the total energy $E$ as a function of cubic gain $\varepsilon$ for families of the fundamental solitons with $S=0$ and rhombic vortices with $S=1$ for zero and nonzero values of strength $p$ of the transverse grating. The shape of the fundamental solitons for different values of $p$ is displayed
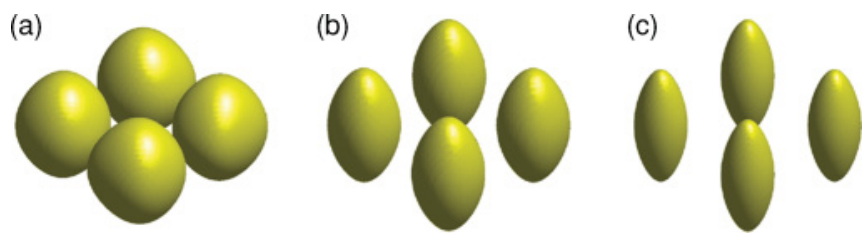

FIG. 3. (Color online) The same as in Fig. 2, but for rhombic vortices with $S=1$ : (a) $p=0.25, \varepsilon=1.9$; (b) $p=1, \varepsilon=1.7$, and (c) $p=4, \varepsilon=1.8$. 


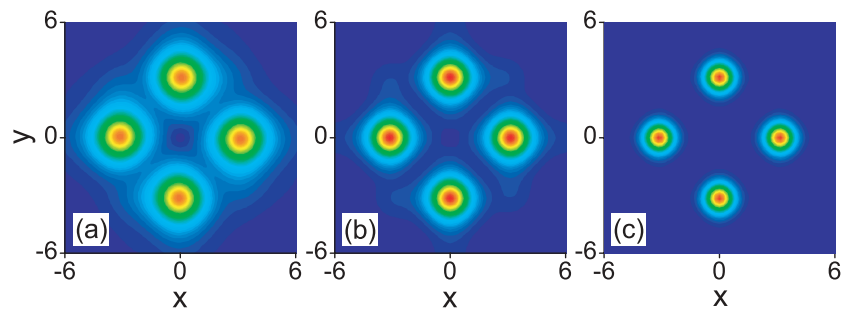

FIG. 4. (Color online) The transverse distribution of the local field amplitude $|U(x, y, 0)|$ in the rhombic vortices shown in Fig. 3.

by means of isosurface plots of total intensity $|U(x, y, t)|^{2}$ in Fig. 2. Similarly, in Figs. 3 and 4 we show the shapes of rhombus-shaped vortices for three representative values of parameter pairs $(p, \varepsilon)$. These figures explain why the relation between the energies of the compound vortices and fundamental solitons $E_{\mathrm{vort}} \approx 4 E_{\mathrm{sol}}$ holds in strong lattices, but not in weaker ones (see Fig. 1) in which the vortex' constituents are not well separated [see Figs. 3(a) and 4(a)]. The families of fundamental solitons shown in Fig. 1(a) are entirely stable (i.e., the solitons feature self-healing) restoring their stationary shape after the addition of random perturbations. The stability of fundamental solitons in the present model is not surprising as they are stable in both the $2 \mathrm{D}$ [16] and 3D [22] versions of Eq. (2) without the lattice potential $(p=0)$; unlike localized vortices, which cannot be stable in either case. Nevertheless, fundamental 3D solitons in the CQ CGL equation with the lattice potential were not studied before, therefore we report here basic results for them-in particular, for comparison with localized vortices. As concerns the stationary rhombic vortices presented by dependences $E=E(\varepsilon)$ in Fig. 1(b), they were generated by direct simulations of Eq. (2), hence they are robust objects in this sense. The grating's potential prevents the splitting of the vortex in the transverse plane. As shown in Ref. [30], this is sufficient for the stabilization of the compound vortices in the 2D counterpart of the present model, which represents spatial vortical solitons. However, in the 3D setting the spatiotemporal vortex complexes develop instability against separation of their constituents in the temporal direction as shown in Fig. 5. This figure demonstrates that the vortex splits into two pairs of fundamental solitons that later split into individual solitons. Nevertheless, Fig. 5 (as well as the results of many other runs of simulations) demonstrates quasistabilization of the vortex solitons with the increase of the grating's strength $p$. Indeed, from Fig. 5 the splitting distance for $p=1, z_{\text {split }} \simeq 100$ may be estimated as tantamount to $\sim 10$ soliton's dispersion lengths,
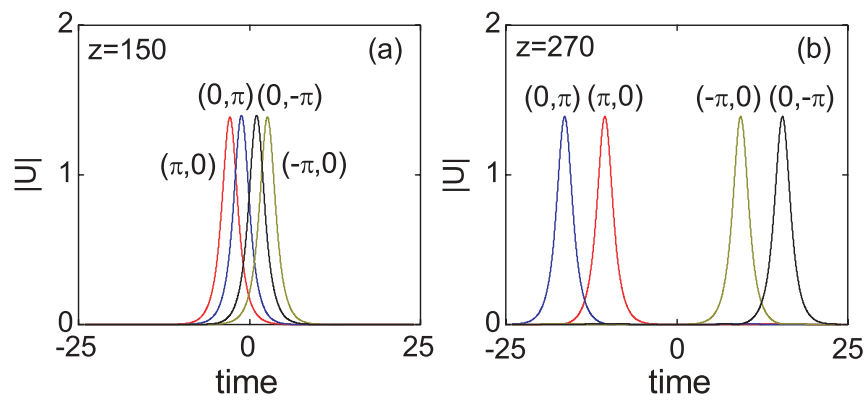

FIG. 5. (Color online) Temporal profiles of the four constituents of an unstable rhombic vortex: (a) at $z=150$; (b) at $z=270$, for $p=1$ and $\varepsilon=1.7$. Labels attached to the plots refer to coordinates of the pulses in the $(x, y)$ plane.

with the temporal width of each constituent being $T \sim 2$. With the increase of the lattice strength to $p=4$, the distance of the stable transmission increases to $z \gtrsim 1500$, which exceeds a meter in physical units (i.e., this vortex will be a completely stable object in the experiment). In this relation, it is relevant to mention a general fact that various complex structures supported by lattice models-first of all, discrete vortex solitons-are stable up to a certain critical value of the intersite coupling constant [34]. In the present case, the latter is tantamount to the stability above a certain minimum value of $p$.

In conclusion, we investigate families of fundamental and vortical spatiotemporal dissipative solitons in the framework of the 3D CGL equation with the CQ nonlinearity, periodic potential in the transverse plane, and anomalous GVD in the temporal direction. The model applies to the description of bulk optical media with the combination of gain and saturable absorption. Solitons of both types were readily found as attractors by direct simulations of the CQ CGL equation. The fundamental solitons are stable against strong random perturbations while the vortices, built as rhombus-shaped complexes of four fundamental solitons with phase shifts $\pi / 2$ between them, may be split by perturbations in the temporal direction. Nevertheless, a sufficiently strong periodic potential inhibits the splitting of the vortices making them practically stable objects.

This work was supported in part by the Deutsche Forschungsgemeinschaft, Bonn and by the Romanian Ministry of Education and Research (Grant No. IDEI-497/2009). H.L. and B.A.M. appreciate the support provided by a joint grant from the Israeli-French High Council for Scientific and Technological Research on the topic of "Nonlinear spatiotemporal photonics in bundled arrays of waveguides."
[1] I. S. Aranson and L. Kramer, Rev. Mod. Phys. 74, 99 (2002); P. Mandel and M. Tlidi, J. Opt. B: Quantum Semiclass. Opt. 6, R60 (2004); B. A. Malomed, in Encyclopedia of Nonlinear Science, edited by A. Scott (Routledge, New York, 2005), p. 157.

[2] N. N. Rosanov, Spatial Hysteresis and Optical Patterns (Springer, Berlin, 2002).
[3] N. Akhmediev and A. Ankiewicz (Eds.), Dissipative Solitons: From Optics to Biology and Medicine, Lect. Notes Phys. 751 (Springer, Berlin, 2008).

[4] S. Barland et al., Nature (London) 419, 699 (2002); Z. Bakonyi et al., J. Opt. Soc. Am. B 19, 487 (2002); E. A. Ultanir, G. I. Stegeman, D. Michaelis, C. H. Lange, and F. Lederer, Phys. Rev. Lett. 90, 253903 (2003); N. N. Rosanov, S. V. Fedorov, and 
A. N. Shatsev, ibid. 95, 053903 (2005); N. Veretenov and M. Tlidi, Phys. Rev. A 80, 023822 (2009).

[5] F. Smektala et al., J. Non-Cryst. Solids 274, 232 (2000); G. Boudebs et al., Opt. Commun. 219, 427 (2003).

[6] C. Zhan et al., J. Opt. Soc. Am. B 19, 369 (2002).

[7] G. S. Agarwal and S. Dutta Gupta, Phys. Rev. A 38, 5678 (1988); E. L. Falcão-Filho et al., J. Opt. Soc. Am. B 24, 2948 (2007).

[8] R. A. Ganeev et al., J. Opt. A: Pure Appl. Opt. 6, 282 (2004).

[9] B. Gu et al., Appl. Phys. Lett. 95, 041114 (2009).

[10] K. Dolgaleva, H. Shin, and R. W. Boyd, Phys. Rev. Lett. 103, 113902 (2009).

[11] B. A. Malomed, Physica D 29, 155 (1987); S. Fauve and O. Thual, Phys. Rev. Lett. 64, 282 (1990); W. van Saarloos and P. C. Hohenberg, ibid. 64, 749 (1990); V. Hakim et al., Europhys. Lett. 11, 19 (1990); B. A. Malomed and A. A. Nepomnyashchy, Phys. Rev. A 42, 6009 (1990); P. Marcq et al., Physica D 73, 305 (1994); N. Akhmediev and V. V. Afanasjev, Phys. Rev. Lett. 75, 2320 (1995); H. R. Brand and R. J. Deissler, ibid. 63, 2801 (1989); J. M. Soto-Crespo, N. Akhmediev, and A. Ankiewicz, ibid. 85, 2937 (2000); H. Leblond, A. Komarov, M. Salhi, A. Haboucha, and F. Sanchez, J. Opt. A 8, 319 (2006); W. H. Renninger, A. Chong, and F. W. Wise, Phys. Rev. A 77, 023814 (2008).

[12] B. A. Malomed et al., J. Opt. B: Quantum Semiclass. Opt. 7, R53 (2005).

[13] A. S. Desyatnikov, Yu. Kivshar, and L.Torner, Prog. Opt. 47, 291 (2005).

[14] A. Desyatnikov, A. Maimistov, and B. Malomed, Phys. Rev. E 61, 3107 (2000); D. Mihalache, D. Mazilu, L. C. Crasovan, B. A. Malomed, and F. Lederer, ibid. 61, 7142 (2000); Phys. Rev. Lett. 88, 073902 (2002); Phys. Rev. E 66, 016613 (2002).

[15] V. I. Petviashvili and A. M. Sergeev, Dokl. Akad. Nauk SSSR 276, 1380 (1984) [Sov. Phys. Dokl. 29, 493 (1984)].

[16] L.-C. Crasovan, B. A. Malomed, and D. Mihalache, Phys. Rev. E 63, 016605 (2001); Phys. Lett. A289, 59 (2001).

[17] P. Grelu et al., Opt. Express 13, 9352 (2005).

[18] J. M. Soto-Crespo et al., Opt. Express 14, 4013 (2006).

[19] V. Skarka and N. B. Aleksić, Phys. Rev. Lett. 96, 013903 (2006).
[20] N. Akhmediev et al., Chaos 17, 037112 (2007).

[21] J. M. Soto-Crespo, N. Akhmediev, and P. Grelu, Phys. Rev. E 74, 046612 (2006).

[22] D. Mihalache et al., Phys. Rev. Lett. 97, 073904 (2006); D. Mihalache, D. Mazilu, F. Lederer, H. Leblond, and B. A. Malomed, Phys. Rev. A 75, 033811 (2007); 76, 045803 (2007).

[23] M. Soljačić, S. Sears, and M. Segev, Phys. Rev. Lett. 81, 4851 (1998).

[24] A. S. Desyatnikov and Y. S. Kivshar, Phys. Rev. Lett. 88, 053901 (2002).

[25] Y. V. Kartashov, L. C. Crasovan, D. Mihalache, and L. Torner, Phys. Rev. Lett. 89, 273902 (2002); L.-C. Crasovan et al., Phys. Rev. E 66, 036612 (2002); 67, 046610 (2003); D. Mihalache et al., ibid. 68, 046612 (2003); J. Opt. B: Quantum Semiclass. Opt. 6, S333 (2004).

[26] D. V. Skryabin and A. G. Vladimirov, Phys. Rev. Lett. 89, 044101 (2002).

[27] W. P. Zhong et al., J. Phys. B 41, 025402 (2008); Y. Q. Wang and Q. Guo, Chin. Phys. B 17, 2527 (2008); M. M. Petroski et al., Opt. Commun. 279, 196 (2007); A. Fratalocchi, A. Piccardi, M. Peccianti, and G. Assanto, Phys. Rev. A 75, 063835 (2007); D. Buccoliero, A. S. Desyatnikov, W. Krolikowski, and Y. S. Kivshar, Phys. Rev. Lett. 98, 053901 (2007); Y. J. He, B. A. Malomed, D. Mihalache, and H. Z. Wang, Phys. Rev. A 77, 043826 (2008).

[28] Y. J. He et al., Opt. Express 15, 17502 (2007).

[29] D. Mihalache, D. Mazilu, F. Lederer, H. Leblond, and B. A. Malomed, Phys. Rev. A 77, 033817 (2008); Phys. Rev. E 78, 056601 (2008); Eur. Phys. J. Special Topics 173, 245 (2009).

[30] H. Leblond, B. A. Malomed, and D. Mihalache, Phys. Rev. A 80, 033835 (2009).

[31] J. Lega, J. V. Moloney, and A. C. Newell, Phys. Rev. Lett. 73, 2978 (1994); Physica D 83, 478 (1995).

[32] R. C. Alferness, in Guided-Wave Optoelectronics, edited by T. Tamir (Springer-Verlag, New York, 1988), pp. 145-210.

[33] J. W. Fleischer et al., Nature (London) 422, 147 (2003).

[34] B. A. Malomed and P. G. Kevrekidis, Phys. Rev. E 64, 026601 (2001). 